\title{
INIBIÇÃO À CORROSÃO DE AÇO-CARBONO EM MEIO SALINO PELO HETEROCICLO ISATINA VEICULADO EM MICROEMULSÕES
}

\author{
Ádna Dornelles Nicácio de Souza ${ }^{a}$, Cátia Guaraciara Fernandes Teixeira Rossia ${ }^{\mathrm{a}}$, Tereza Neuma de Castro Dantas ${ }^{\mathrm{a}}$, Miguel \\ Ângelo Fonseca de Souza a, Djalan França de Lima a , Fabrício Gava Menezes ${ }^{\mathrm{a}}$ e Maria Aparecida Medeiros Maciela, ${ }^{\mathrm{b}, *, \ldots}$ \\ anstituto de Química, Universidade Federal do Rio Grande do Norte, Campus Universitário, Lagoa Nova, 59072-970 Natal - RN, \\ Brasil \\ ${ }^{b}$ Universidade Potiguar Laureate International Universities, Campus Salgado Filho, Natal - RN, Brasil
}

Recebido em 18/12/2016; aceito em 16/03/2017; publicado na web em 15/05/2017

\begin{abstract}
EFFICIENCY OF THE HETEROCYCLE ISATIN LOADED IN MICROEMULSIONS AS CORROSION INHIBITOR FOR MILD STEEL IN SALINE MEDIUM. Polar microemulsion systems (SME) based on saponified coconut oil (OCS) as surfactant were prepared in order to increase the solubility of the heterocycle isatin (IST) aiming at its application as corrosion inhibitor against AISI 1020 mild steel, in saline medium. The adsorptions phenomena of the tested SME systems (SME-OCS1-IST and SME-OCS2-IST) in the liquid/gas interface were evaluated by surface tension measurements which do not show significant differences in the presence of ion $\mathrm{Cl}^{-}$. The free energy values of the micellization proved the occurrence of spontaneous process. The corrosion inhibition effectiveness of SME-OCS2, SME-OCS1-IST and SME-OCS2-IST samples was evaluated in $0.5 \%$ aqueous NaCl using voltammetry linear scan method (LPR). The maximum efficiency findings were 73.2\% for SME-OCS2, 88.2\% for SME-OCS2-IST and 97.6\% for SME-OCS1-IST. The spontaneity of the adsorption phenomena was also observed in the liquid/metal interface by analyzes of the adsorption free energy. In addition, since computational calculations have been found as a very relevant tool in the corrosion field, a theoretical study was performed in attempt to give support to the observed experimental results, in which B3LYP and 6-311++G(d,p) were applied aiming at investigation of geometry, electronic density aspects, HOMO-LUMO energy gap, as well as the binding between isatin and metal surface.
\end{abstract}

Keywords: isatin, microemulsion o/w systems; corrosion inhibitor; saline medium; LPR electrochemical methodology; theoretical study using B3LYP and 6-311++G(d,p).

\section{INTRODUÇÃO}

A corrosão acarreta prejuízos financeiros e operacionais significativos, além de danos ambientais e riscos operacionais. Em função de causar deterioração de equipamentos e estruturas metálicas, o fenômeno da corrosão se destaca como um dos maiores problemas das indústrias da atualidade. $\mathrm{O}$ aço-carbono, em função de suas propriedades, simples fabricação e baixo custo, é um dos principais tipos de aço empregados na indústria, no entanto, a exemplo de outros materiais, está sujeito à corrosão por diversos meios. Por esta razão, a demanda, em todo o mundo, por agentes inibidores de corrosão se mostra altamente atrativa para o setor industrial. ${ }^{1-8}$

Várias pesquisas vêm sendo desenvolvidas no sentido de controlar a corrosão. Dentre os métodos mais utilizados, destaca-se o uso de inibidores de corrosão por ação de películas protetoras que se adsorvem às áreas anódicas e catódicas do metal, exercendo controle sobre os processos eletroquímicos que desencadeiam a corrosão. Em função da eficácia de filmes protetores que se formam na interface entre o metal e o meio corrosivo, destaca-se o uso de moléculas orgânicas contendo nitrogênio, oxigênio e enxofre. ${ }^{9-15}$ Esta proteção está vinculada às interações entre heteroátomos presentes em suas estruturas com a superfície do metal, cuja natureza e carga superficial também influenciam diretamente o processo de adsorção.

A isatina (IST) é um composto orgânico heterociclo quem vem sendo amplamente utilizado em pesquisas científicas. Citando apenas um exemplo, Pinto e colaboradores desenvolveram diversos estudos com este composto objetivando incentivar o uso industrial da isatina,

*e-mail: mammaciel@hotmail.com

\#Programa de Pós-graduação em Biotecnologia tendo sido co-autor de uma extensa revisão acerca da química dos derivados de IST que resultou em uma publicação em que se destacam diversas metodologias de síntese e aplicações em diversas áreas. ${ }^{16} \mathrm{~A}$ capacidade da IST na inibição à corrosão de metais (alumínio e aço carbono) danificados pela ação de meios ácidos se encontra reportada em três artigos científicos. ${ }^{17-19}$

O desenvolvimento de produtos capazes de inibir a corrosão proveniente de um meio rico em ânions cloreto, em especial oriundos do ambiente marinho, tem se mostrado uma promissora fonte de pesquisa para indústrias petrolíferas, especialmente por conta das maiores reservas de petróleo estarem localizadas no mar. Um dos principais fatores associados à aplicabilidade de um inibidor de corrosão em meio aquoso neutro ou salino consiste na problemática da solubilidade. Neste contexto, os sistemas coloidais do tipo microemulsão (SME), em decorrência da sua baixa tensão interfacial, baixo custo e facilidade de obtenção, surgem como um meio alternativo para a solubilização e aplicações diversificadas de moléculas orgânicas. ${ }^{20-24}$

Microemulsões podem ser definidas como sistemas termodinamicamente estáveis, isotrópicos, opticamente transparentes, de baixa viscosidade, que se formam a partir de uma solubilização espontânea de dois líquidos imiscíveis (água, óleo) na presença de um tensoativo e, se necessário, um cotensoativo. ${ }^{20-24}$

Com relação ao uso de microemulsão do tipo O/A (sistema polar rico em água), objetivando a disponibilização de compostos orgânicos para aplicação na inibição à corrosão, encontram-se os formulados SME-OCS que contém óleo de coso saponificado (OCS) como tensoativo. Este sistema padrão veiculou satisfatoriamente tiosemicarbazonas e a difenilsemicarbazida que foram avaliadas em meio salino $(0,5 \%$ de $\mathrm{NaCl})$ como inibidores de corrosão de aço carbono AISI 1020, com eficiências entre $84 \%$ e $92 \%$. O tensoativo semissintético 
OCS foi obtido a partir do óleo de coco, cuja escolha se justifica por ser uma fonte abundante subutilizada na região nordeste do Brasil, que tem baixo custo e é de fácil obtenção. ${ }^{23,24}$

No presente trabalho encontra-se relatada a ação anticorrosiva do heterociclo isatina (IST) veiculado em sistemas coloidais do tipo O/A (óleo em água) contendo o tensoativo biodegradável OCS. A técnica de voltametria de varredura linear (método LPR) em aço carbono AISI-1020, no meio corrosivo salino $(0,5 \%$ de $\mathrm{NaCl})$, bem como estudos de adsorção na interfaces líquido/sólido e líquido/ gás, foram utilizados para os sistemas SME-OCS2, SME-OCS1IST e SME-OCS2-IST. Cálculos computacionais foram realizados objetivando a avaliação teórica do inibidor IST e seu mecanismo de ação na inibição à corrosão.

\section{PARTE EXPERIMENTAL}

\section{Obtenção do óleo de coco saponificado (OCS)}

O tensoativo OCS foi obtido a partir da saponificação do óleo de coco de acordo com metodologia previamente reportada. ${ }^{23}$ Uma solução contendo $20,89 \mathrm{~g}$ de $\mathrm{NaOH}$ dissolvidos em $80 \mathrm{~mL}$ de água destilada foi adicionada a $100 \mathrm{~g}$ de óleo de coco. A essa mistura foram adicionados $300 \mathrm{~mL}$ de álcool etílico e o sistema reacional foi mantido sob refluxo por $2 \mathrm{~h}$. Após esse tempo, o álcool foi eliminado por rota evaporação, sendo o produto resultante seco a $40{ }^{\circ} \mathrm{C}$.

Os índices de acidez, de iodo e de saponificação do óleo de coco foram determinados de acordo com as metodologias apresentadas no Material Suplementar.

\section{Obtenção dos sistemas microemulsionados}

O sistema microemulsionado foi preparado com base na metodologia de titulações e frações mássicas em diagrama pseudoternário. ${ }^{23,24}$ Especificamente, utilizou-se óleo de coco saponificado (OCS) como tensoativo (T), butanol como cotensoativo (C), querosene como fase oleosa (FO) e água destilada (fase aquosa, FA). $\mathrm{O}$ procedimento utilizado para se obter a região de microemulsão baseia-se no método que envolve a determinação dos pontos de solubilidade máximas da matéria ativa (no caso, C/T na razão 1:1) nas fases FO e FA por meio de titulações mássicas, para produzir sistema coloidal do tipo O/A (sistema rico em água). O sistema menos polar SME-OCS1 (20\% de OCS, 20\% de butanol, $5 \%$ de FO e $55 \%$ de FA), bem como o sistema SME-OCS2, de maior polaridade ( $12,5 \%$ de OCS, $12,5 \%$ de butanol, $5 \%$ de FO e $70 \%$ de FA), foram avaliados pela obtenção de diagramas pseudoternários, que possibilitaram estabelecer as composições totais destes sistemas. A análise reológica e a medida de tensão superficial de cada sistema foram realizadas conforme descrito no Material Suplementar, estando de acordo com metodologia reportada. ${ }^{23,24}$

\section{Estudo da solubilização da isatina (IST) nos sistemas microemulsionados SME-OCS}

As concentrações de IST incorporadas nos sistemas SME-OCS1 e SME-OCS2 foram determinadas via espectroscopia UV-Vis, no espectrômetro Genesys 10-UV/Vis, Thermo Electron Corporation. Os estudos foram realizados em uma faixa de comprimento de onda entre $200 \mathrm{~nm}$ - $400 \mathrm{~nm}$, com quantificação de veiculação da IST através das absorbâncias em $\lambda=229 \mathrm{~nm}$ (SME-OCS1) e $217 \mathrm{~nm}$ (SME-OCS2). As absorbâncias destas microemulsões e dos sistemas contendo IST (SME-OCS1-IST e SME-OCS2-IST) foram medidas após sucessivas diluições em metanol. Todas as medidas foram realizadas em triplicata.

\section{Medidas de eficiência de inibição à corrosão}

As medidas de eficiência de inibição à corrosão dos sistemas isentos de IST (SME-OCS1 e SME-OCS2), bem como dos formulados SME-OCS1-IST e SME-OCS2-IST (sistemas contendo isatina), foram realizadas em meio aquoso salino $(\mathrm{NaCl} 0,5 \%)$ pela técnica de voltametria de varredura linear. Apenas para o sistema SME-OCS1 foi realizada, nesta mesma metodologia, avaliação preliminar. ${ }^{23} \mathrm{Os}$ ensaios foram realizados em célula clássica de três eletrodos, com capacidade para $30 \mathrm{~mL}$ de solução, formada por um eletrodo de referência de $\mathrm{Ag} / \mathrm{AgCl}\left(3,0 \mathrm{~mol} \mathrm{~L}^{-1} \mathrm{KCl}\right)$, o contraeletrodo de grafite e o eletrodo de trabalho de aço carbono AISI-1020, com composição química $0,18 \%-0,23 \%$ de carbono, $0,30 \%-0,60 \%$ de manganês, $0,030 \%$ de fósforo, $0,050 \%$ de enxofre, $0,0005 \%-0,003 \%$ de boro e $0,20 \%$ de cobre, com área superficial de $0,273 \mathrm{~cm}^{2}$, tendo sido polido até o espelhamento. Utilizou-se um potenciostato/galvanostato de modelo $302 \mathrm{~N}$ da AUTOLAB PGSTAT para medidas de inibição à corrosão, à temperatura ambiente de $298 \mathrm{~K}$. Os dados obtidos da análise de resistência polarização linear (LPR) foram analisadas utilizando o software GPES da AUTOLAB, versão 4.9, obtendo-se as curvas de Tafel.

Para obtenção das curvas de polarização, o eletrodo de trabalho, os eletrodos de referência e contraeletrodo foram imersos em solução aquosa salina $(\mathrm{NaCl} 0,5)$ durante 15 minutos para estabelecer um potencial de circuito aberto de estado estacionário $\left(\mathrm{E}_{\mathrm{ocp}}\right)$. Após a medição (Eocp), potencial constante em torno de - $500 \mathrm{mV}$, as análises eletroquímicas (LPR) foram conduzidas a uma taxa de varredura de $1 \mathrm{mVs}^{-1}$. Na sequência, os estudos de polarização anódica e catódica foram conduzidos com variação do potencial de Eocp na faixa -500 $\mathrm{mV}$ a $500 \mathrm{mV}$. Nestas condições experimentais, a concentração do inibidor variou de acordo com os valores mostrados na Tabela 1.

Tabela 1. Percentual das concentrações de IST veiculada em cada sistema SME-OCS em função do volume da solução salina $(\mathrm{NaCl} 0.5 \%)$

\begin{tabular}{ccc}
\hline $\mathrm{V}_{\text {NaCl }}(\mathrm{mL})$ & $\mathrm{V}_{\text {INIBIDOR }}(\mathrm{mL})$ & $\mathrm{C}_{\text {INIBIDOR }}(\%)$ \\
\hline 40 & 0 & 0 \\
36 & 4 & 10 \\
32 & 8 & 20 \\
28 & 12 & 30 \\
24 & 16 & 40 \\
20 & 20 & 50 \\
16 & 24 & 60 \\
12 & 28 & 70 \\
8 & 32 & 80 \\
4 & 36 & 90 \\
0 & 40 & 100 \\
\hline
\end{tabular}

Os segmentos lineares anódicos e catódicos das curvas de Tafel foram extrapolados no ponto de interseção, de acordo com os dados de potencial de corrosão $\left(\mathrm{E}_{\text {corr }}\right)$ e de densidade de corrente de corrosão $\left(i_{\text {corr }}\right)$. As eficiências de inibição, em diferentes concentrações de inibidor, foram calculadas utilizando a Equação 1.

$$
\text { Eficiência de inibição }\left(\eta_{P}\right)=\left[\left(\frac{i_{0}-i}{i_{0}}\right)\right] \times 100 \%
$$

em que "i" é a densidade de corrente para as amostras analisadas, e " $\mathrm{i}_{\mathrm{o}}$ " é a densidade de corrente da amostra contendo apenas $\mathrm{NaCl}$ (branco). 


\section{Estudo computacional}

Os parâmetros estruturais e eletrônicos para a IST foram obtidos em fase gasosa utilizando o Programa Gaussian, ${ }^{25}$ através do método B3LYP, ${ }^{26}$ combinado com o conjunto de bases $6-311++G(d, p)$. As cargas atômicas foram calculadas de acordo com o método Chelpg. ${ }^{27}$ A partir do estudo computacional foi possível obter os valores teóricos de momento de dipolo $(\mu)$, assim como as energias do orbital molecular ocupado de mais alta energia $\left(\mathrm{E}_{\mathrm{HOMO}}\right)$ e do orbital molecular não ocupado de mais baixa energia $\left(\mathrm{E}_{\mathrm{LUMO}}\right)$. A afinidade eletrônica $(\mathrm{A})$ e o potencial de ionização (I) foram obtidos a partir das Equações 2 e 3, sendo que os mesmos foram utilizados para obtenção dos valores de eletronegatividade absoluta $(\chi)$ e dureza global $(\eta)$ segundo as Equações 4 e $5 .{ }^{28}$ A fração de elétrons transferidos $(\Delta N)$ foi calculada através da Equação 6, considerando as eletronegatividades absolutas do átomo de ferro e da IST sendo, respectivamente, $\chi_{\mathrm{Fe}}$ e $\chi_{\text {inh }}$, e as durezas absolutas do metal e do inibidor como sendo $\chi_{\mathrm{Fe}}$ and $\chi_{\text {inh }}$, respectivamente. Neste contexto, $\gamma=(\mathrm{I}-\mathrm{A}) / 2$. Foram considerados os valores teóricos de $\chi_{\mathrm{Fe}}=7 \mathrm{eV} / \mathrm{mol}$ e $\gamma_{\mathrm{Fe}}=0$ com base na aceitação de que I=A para um "bulk' metálico. ${ }^{28}$

$$
\begin{gathered}
\mathrm{A}=-\mathrm{E}_{\mathrm{LUMO}} \\
\mathrm{I}=-\mathrm{E}_{\mathrm{HOMO}} \\
\chi=(\mathrm{I}+\mathrm{A}) / 2 \\
\eta=\left(\mathrm{E}_{\mathrm{LUMO}}-\mathrm{E}_{\mathrm{HOMO}}\right) / 2 \\
\Delta \mathrm{N}=\left(\chi_{\mathrm{Fe}-} \chi_{\mathrm{IST}}\right) / 2\left(\gamma_{\mathrm{Fe}}+\gamma_{\mathrm{IST}}\right)
\end{gathered}
$$

\section{RESULTADOS E DISCUSSÃO}

\section{Análises fisico-químicas do óleo vegetal}

O tensoativo óleo de coco saponificado (OCS) foi obtido a partir da fonte vegetal óleo de coco que sofreu processo de saponificação de acordo com metodologia previamente descrita. ${ }^{23}$ As análises físico-químicas do óleo vegetal (Tabela 2) mostraram índices oleoquímicos compatíveis com os valores observados por Rossi e colaboradores. ${ }^{23}$

Tabela 2. Análises físico-químicas do óleo de coco

\begin{tabular}{lc}
\hline Índices & Valores obtidos \\
\hline Índice de acidez & 12,77 \\
Índice de iodo & 7,66 \\
Índice de saponificação & 228,34 \\
\hline
\end{tabular}

\section{Obtenção do sistema microemulsionado a base do tensoativo OCS}

A hidrólise alcalina do óleo de coco resultou na formação do tensoativo OCS que foi utilizado para preparar a microemulsão padrão SME-OCS que contém, em proporções diferenciadas para cada sistema (SME-OCS1 e SME-OC2), os seguintes componentes: OCS e butanol na razão $\mathrm{C} / \mathrm{T}=1$, querosene como fase óleo e água destilada, como mostrado na Tabela 3. A partir do diagrama pseudoternário (Figura 1) do sistema padrão (SME-OCS) foram escolhidos dois pontos na região de Winsor IV, que possibilitaram a obtenção dos sistemas derivativos SME-OCS1 e SME-OC2.

A justificativa para se trabalhar com uma composição na região Winsor IV consiste no fato de se ter uma maior área de microemulsão com pontos de solubilidade máximos da matéria ativa C/T (co-tensoativo/tensoativo, no caso do sistema padrão SME-OCS, o co-tensoativo utilizado foi o butanol). Para o sistema padrão SME-OCS há formação de região WIV com particulados em suspensão (WIV + S) que correspondem ao OCS não solubilizado. Após sucessivas diluições em água, ambos sistemas SME-OCS1 (40\% de C/T, 5\% de fase óleo e $55 \%$ de $\mathrm{H}_{2} \mathrm{O}$ ) e SME-OCS2 (25\% de C/T, 5\% de fase óleo e $70 \%$ de $\mathrm{H}_{2} \mathrm{O}$ ) se apresentaram estáveis, ou seja, não há quebra da região de microemulsão WIV.

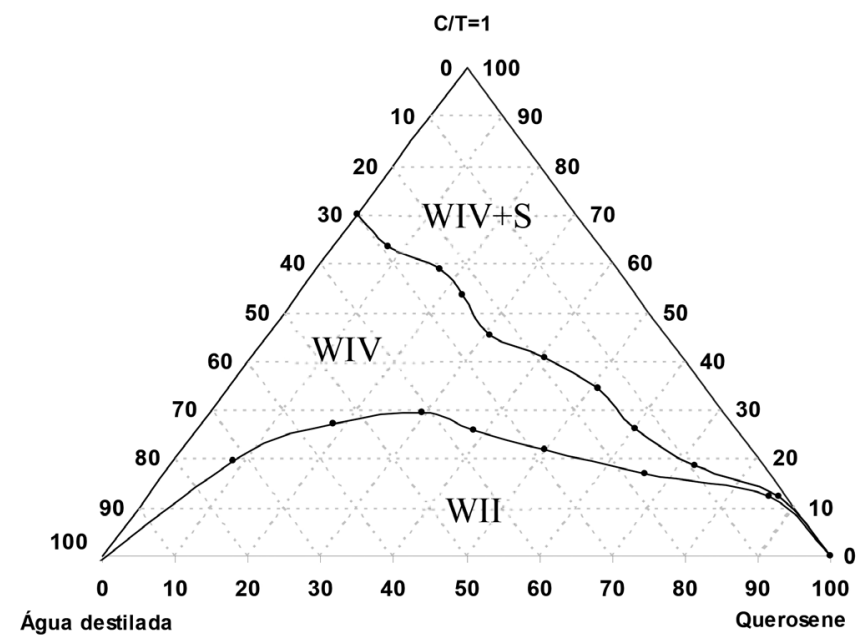

Figura 1. Diagrama pseudoternário do sistema microemulsionado SME-OCS

Tabela 3. Composição dos sistemas microemulsionados contendo OCS

\begin{tabular}{lcccc}
\hline SISTEMA & OCS $(\%)$ & $\begin{array}{c}\text { Butanol } \\
(\%)\end{array}$ & $\begin{array}{c}\text { Querosene } \\
(\%)\end{array}$ & $\begin{array}{c}\text { Água } \\
\text { Destilada }(\%)\end{array}$ \\
\hline SME-OCS1 & 20,0 & 20,0 & 5 & 55 \\
SME-OCS2 & 12,5 & 12,5 & 5 & 70 \\
\hline
\end{tabular}

Quantificação de isatina solubilizada nos sistemas microemulsionados

A quantificação de IST solubilizada nos sistemas SME-OCS1 e SME-OCS2 foi determinada por espectroscopia na região do UV-Vis, tendo apresentado solubilidade máxima na microemulsão de menor polaridade (SME-OCS1), como mostrado na Tabela 4. Observou-se que a diferença de polaridade entre os sistemas SME-OCS1 e SME-OCS2 influencia na solubilização do heterociclo isatina (IST), que após veiculação nestas microemulsões foi avaliado como inibidor de corrosão.

Considerando a importância da microemulsão padrão SME-OCS como sistema veiculador de compostos orgânicos ${ }^{23,24} \mathrm{e}$, ainda, em função das variações de concentrações do tensoativo OCS, água e solubilidades máximas da IST (4,64 mg IST/mL SME-OCS1 e 0,99 mg IST/mL SME-OCS2), optou-se por avaliar a eficiência de inibição à corrosão da IST veiculada nos sistemas SME-OCS1-IST e SME-OCS2-IST, em percentuais significantemente reduzidos $\left(3,71 \times 10^{-2} \mathrm{mg}-7,929 \times 10^{-3} \mathrm{mg}\right)$ (Tabela 6).

Tabela 4. Solubilidade de IST em cada sistema SME-OCS

\begin{tabular}{lccc}
\hline SISTEMA & $\lambda_{\max }$ & Solubilidade em $\mathrm{mg} / \mathrm{mL}^{*}$ & Solubilidade (\%) \\
\hline SME-OCS1 & 229 & $4,640 \pm 0,000$ & 19 \\
SME-OCS2 & 217 & $0,990 \pm 0,136$ & 8 \\
\hline
\end{tabular}

*Os valores correspondem à média em triplicata.

\section{Medidas de viscosidade e de tensão superficial dos sistemas} microemulsionados

As medidas de viscosidade dos sistemas SME-OCS1, SMEOCS2, SME-OCS1-IST e SME-OCS2-IST foram obtidas com 
variação de temperatura $\left(25{ }^{\circ} \mathrm{C}-60{ }^{\circ} \mathrm{C}\right)$. Os gráficos de tensão de cisalhamento em função da taxa de cisalhamento estão apresentados nas Figuras 2a-d. Observou-se que todos os sistemas apresentam viscosidade constante a uma temperatura e pressão constantes. Portanto, a viscosidade independe da taxa de cisalhamento e as microemulsões apresentam comportamento de fluido Newtoniano.

O estudo de adsorção na interface líquido-gás e o comportamento micelar dos sistemas SME-OCS1-IST e SME-OCS2-IST foram avaliados a partir de medidas de tensão superficial. A concentração micelar crítica (CMC) de cada sistema foi determinada em meio neutro e salino $0,5 \%$ de $\mathrm{NaCl}$. As Figuras $3 \mathrm{a}$ e $3 \mathrm{~b}$ mostram as curvas de determinação da CMC através dos valores da tensão superficial em função da concentração do tensoativo OCS nos sistemas contendo IST (SME-OCS1-IST e SME-OCS2-IST, respectivamente). De acordo com os resultados observados sugere-se que a presença do íon $\left(\mathrm{Cl}^{-}\right)$ não interfere na formação das micelas.

(a)

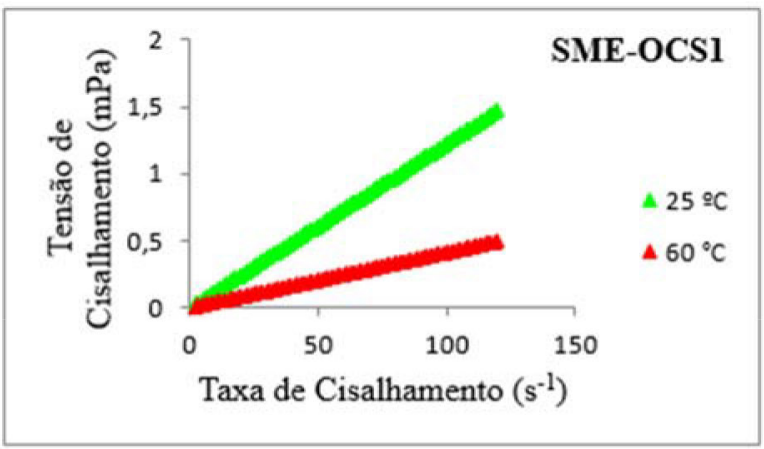

(b)

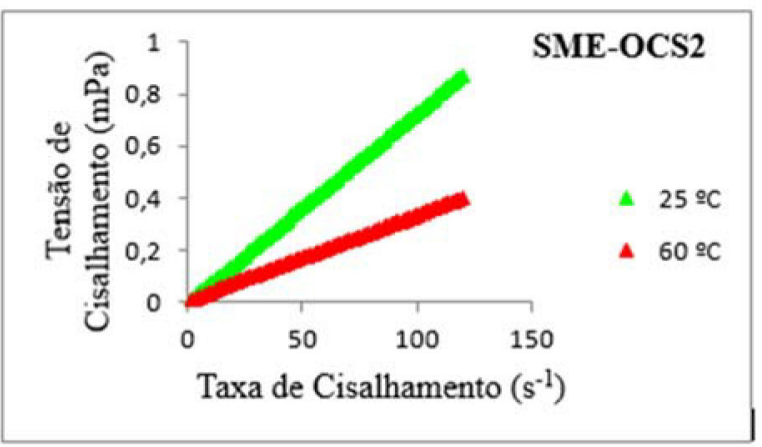

A Tabela 5 apresenta os parâmetros fisico-químicos para o fenômeno de adsorção dos sistemas microemulsionados preparados com fase água neutra (SME-OCS1-IST- $\mathrm{H}_{2} \mathrm{O}$ e SME-OCS2-IST-H ${ }_{2} \mathrm{O}$ ) e salina (SME-OCS1-IST-NaCl 0,5\% e SME-OCS2-IST-NaCl 0,5\%). Verificou-se que a energia livre de micelização $\left(\Delta \mathrm{G}_{\mathrm{mic}}\right)$ é negativa, indicando a espontaneidade da formação das micelas. Este resultado sugere que a adsorção ocorre na interface líquido-gás das microgotículas de cada sistema SME, no entanto, em meio salino observa-se aumento dos valores de $\Delta \mathrm{G}_{\text {mic }}$ para ambos parâmetros [excesso superficial $(\Gamma)$ e área superficial], ou seja, a área ocupada por cada molécula torna-se maior já que os valores deste parâmetro mostraram que há uma ampla área de adsorção na interface $\left(\AA^{2}\right)$. O aumento dos parâmetros $\Delta \mathrm{G}_{\text {mic }}$ e $\Gamma$ para os sistemas preparados em meio salino pode estar correlacionado com as interações do íon $\mathrm{Cl}^{-}$com o grupo hidrofílico do tensoativo OCS (o íon $\mathrm{Cl}^{-}$diminui a repulsão entre as cabeças polares do tensoativo) (Figura 3).

(c)

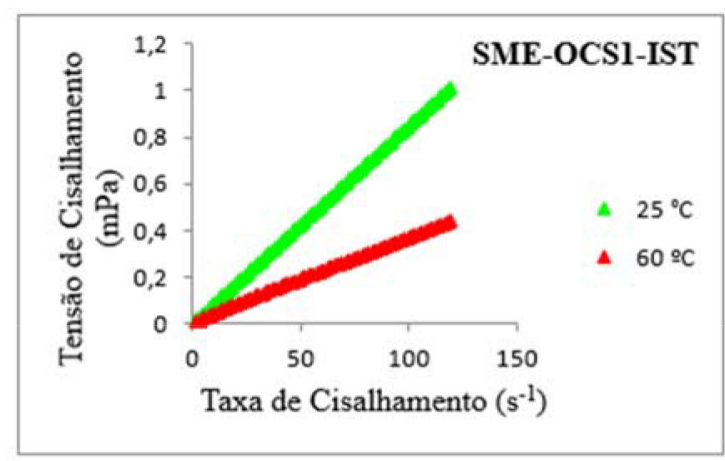

(d)

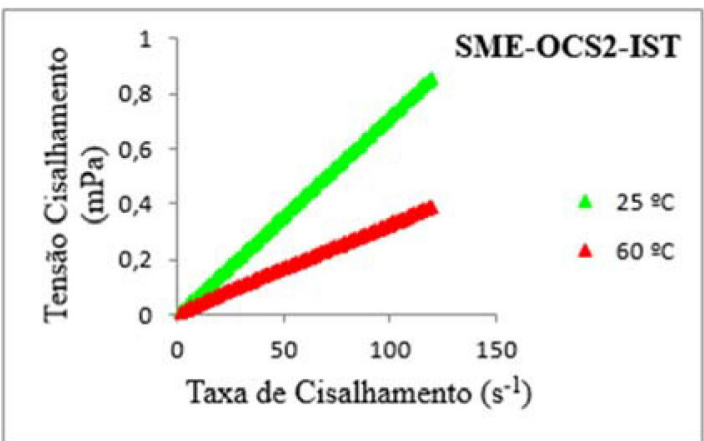

Figura 2. Comportamento reológico dos sistemas microemulsionados: (a) SME-OCS1; (b) SME-OCS2; (c) SME-OCS1-IST; (d) SME-OCS2-IST
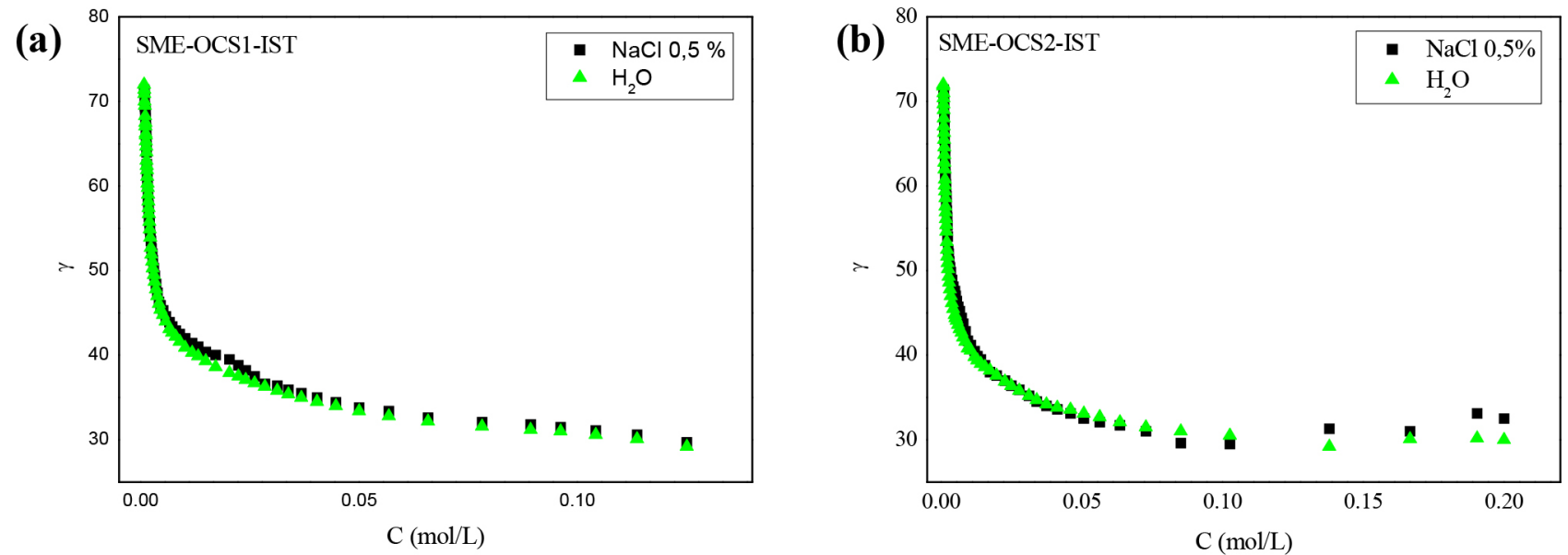

Figura 3. Curvas de determinação da CMC para os sistemas microemulsionados: (a) SME-OCS1-IST; (b) SME-OCS2-IST, preparados em meio aquoso salino $(\mathrm{NaCl} 0,5 \%)$ 
Tabela 5. Parâmetros físico-químicos para SME-OCS-IST preparado em meios aquoso e salino

\begin{tabular}{lcccc}
\hline SISTEMA & $\mathrm{CMC}$ & $\Delta \mathrm{G}_{\text {mic }}(\mathrm{kJ} / \mathrm{mol})$ & $\Gamma$ & $\mathrm{A}\left(\AA^{2}\right)$ \\
\hline SME-OCS1-IST- ${ }_{2} \mathrm{O}$ & 0,0109 & $-10,26$ & $-3.88 \mathrm{E}-06$ & $-42,74$ \\
SME-OCS1-IST-NaCl & 0,0049 & $-12,08$ & $-2.64 \mathrm{E}-06$ & $-62,83$ \\
SME-OCS2-IST- $\mathrm{H}_{2} \mathrm{O}$ & 0,0046 & $-12,22$ & $-3.81 \mathrm{E}-06$ & $-43,61$ \\
SME-OCS2-IST-NaCl & 0,0041 & $-12,48$ & $-3.01 \mathrm{E}-06$ & $-55,24$ \\
\hline
\end{tabular}

\section{Medidas da eficiência de inibição à corrosão}

O estudo da eficiência de inibição à corrosão em aço AISI 1020 foi realizado para os sistemas SME-OCS2 (isento de isatina), SMEOCS1-IST e SME-OCS2-IST. Em trabalhos anteriores, a eficácia de inibição à corrosão do tensoativo OCS livre e microemulsionado (sistema padrão SME-OCS) foram testados pelo método de curvas de polarização linear (método LPR), com eficiências máximas de inibição $63 \%$ e $77 \%$, respectivamente..$^{23}$ Para este sistema, encontra-se reportado que derivados de carbazida, tiossemicarbazida e tiossemicarbazona, solubilizados em baixas concentrações, na microemulsão padrão SME-OCS, apresentaram eficiências máximas de inibição à corrosão significativas $(83 \%$ - 94\%) em meio corrosivo salino. ${ }^{23,24}$
No presente trabalho, a eficiência de inibição à corrosão do heterociclo isatina (IST) solubilizado em baixas concentrações, nos sistemas SME-OCS1 (40\% de C/T) e SME-OCS2 (25\% de C/T), foi avaliada utilizando-se a técnica de curvas de polarização linear. Os cálculos das eficiências máximas de inibição à corrosão foram realizados a partir das curvas de Tafel (obtidas através de curvas LPR), que possibilitaram a obtenção dos valores das correntes (i), que por sua vez forneceram as variações de inibições para os sistemas SME-OCS1-IST e SME-OCS2-IST. Observou-se que o aumento na concentração do inibidor IST $\left(3,71 \times 10^{-2} \mathrm{mg}\right.$, SME-OCS1-IST e 7,92 x 10-3 mg, SME-OCS2-IST) ocasionou diminuição no valor da corrente (Tabela 6), indicando que IST em maiores concentrações retarda mais eficazmente o processo de corrosão. O sistema SMEOCS1-IST apresentou eficácia máxima de inibição $(97,7 \%)$ já que contém na sua composição, maior percentual de OCS (20\%) e menor quantidade de $\mathrm{H}_{2} \mathrm{O}$ (55\%), sendo também o sistema que melhor solubilizou o inibidor IST (19\% de solubilização).

As eficiências máximas de inibição à corrosão do sistema padrão SME-OCS $(77,0 \%)^{23}$ e do sistema SME-OCS2 $(73,2 \%)$ avaliados para o aço carbono AISI 1020, em meio corrosivo salino, (Tabela 7) são correlacionadas ao tensoativo OCS. A incorporação do heterociclo IST causa diferença significante: $97,7 \%$ para SME-OCS1-IST e $88,2 \%$ para SME-OCS2-IST, no combate à corrosão do aço carbono AISI 1020, em meio salino (Tabela 6).

Tabela 6. Eficiências de inibição à corrosão dos sistemas microemulsionados a base de OCS e IST

\begin{tabular}{|c|c|c|c|c|c|c|c|}
\hline SISTEMA & $\mathrm{V}_{\mathrm{NaCl}}(\mathrm{mL})$ & $\mathrm{V}_{\mathrm{SME}}(\mathrm{mL})$ & [SME] $(\%)$ & {$[\mathrm{OCS}](\mathrm{mg})$} & [IST] (mg) & $\mathrm{i}_{\text {corrosão }}$ & $\mathrm{E}(\%)$ \\
\hline \multirow{11}{*}{ SME-OCS2 } & 40 & 0 & 0 & 0,0 & - & 7,02E-06 & 0 \\
\hline & 36 & 4 & 10 & 0,5 & - & 6,06 E-06 & 13,6 \\
\hline & 32 & 8 & 20 & 1,0 & - & 4,86 E-06 & 30,7 \\
\hline & 28 & 12 & 30 & 1,5 & - & 3,31 E-06 & 52,8 \\
\hline & 24 & 16 & 40 & 2,0 & - & 2,96 E-06 & 57,8 \\
\hline & 20 & 20 & 50 & 2,5 & - & 2,57 E-06 & 63,4 \\
\hline & 16 & 24 & 60 & 3,0 & - & $2,25 \mathrm{E}-06$ & 67,9 \\
\hline & 12 & 28 & 70 & 3,5 & - & 2,19 E-06 & 68,8 \\
\hline & 8 & 32 & 80 & 4,0 & - & 2,13 E-06 & 69,6 \\
\hline & 4 & 36 & 90 & 4,5 & - & 2,01 E-06 & 71,3 \\
\hline & 0 & 40 & 100 & 5,0 & - & 1,88 E-06 & 73,2 \\
\hline \multirow{11}{*}{ SME-OCS1-IST } & 40 & 0 & 0 & 0,0 & 0,0 & 7,02E-06 & 0 \\
\hline & 36 & 4 & 10 & 0,8 & $3,71 \mathrm{E}-03$ & 4,96E-06 & 29,3 \\
\hline & 32 & 8 & 20 & 1,6 & $7,42 \mathrm{E}-03$ & $3,64 \mathrm{E}-06$ & 48,2 \\
\hline & 28 & 12 & 30 & 2,0 & $1,11 \mathrm{E}-02$ & $3,11 \mathrm{E}-06$ & 55,7 \\
\hline & 24 & 16 & 40 & 3,2 & $1,48 \mathrm{E}-02$ & 2,61E-06 & 62,9 \\
\hline & 20 & 20 & 50 & 4,0 & $1,86 \mathrm{E}-02$ & $2,06 \mathrm{E}-06$ & 70,7 \\
\hline & 16 & 24 & 60 & 4,8 & $2,23 \mathrm{E}-02$ & $1,63 \mathrm{E}-06$ & 76,7 \\
\hline & 12 & 28 & 70 & 5,6 & $2,60 \mathrm{E}-02$ & $1,06 \mathrm{E}-06$ & 84,9 \\
\hline & 8 & 32 & 80 & 6,4 & 2,97E-02 & $5,13 \mathrm{E}-07$ & 92,7 \\
\hline & 4 & 36 & 90 & 7,2 & $3,34 \mathrm{E}-02$ & $3,42 \mathrm{E}-07$ & 95,1 \\
\hline & 0 & 40 & 100 & 8,0 & 3,71E-02 & $1,64 \mathrm{E}-07$ & 97,7 \\
\hline \multirow{11}{*}{ SME-OCS2-IST } & 40 & 0 & 0 & 0,0 & 0,0 & $7,02 \mathrm{E}-06$ & 0 \\
\hline & 36 & 4 & 10 & 0,5 & 7,92E-04 & $5,48 \mathrm{E}-06$ & 21,8 \\
\hline & 32 & 8 & 20 & 1,0 & $1,58 \mathrm{E}-03$ & $3,30 \mathrm{E}-06$ & 52,9 \\
\hline & 28 & 12 & 30 & 1,5 & $2,38 \mathrm{E}-03$ & $2,78 \mathrm{E}-06$ & 60,8 \\
\hline & 24 & 16 & 40 & 2,0 & $3,17 \mathrm{E}-03$ & $2,36 \mathrm{E}-06$ & 66,4 \\
\hline & 20 & 20 & 50 & 2,5 & $3,96 \mathrm{E}-03$ & $2,13 \mathrm{E}-06$ & 69,7 \\
\hline & 16 & 24 & 60 & 3,0 & $4,75 \mathrm{E}-03$ & $1,70 \mathrm{E}-06$ & 75,7 \\
\hline & 12 & 28 & 70 & 3,5 & $5,54 \mathrm{E}-03$ & $1,34 \mathrm{E}-06$ & 80,9 \\
\hline & 8 & 32 & 80 & 4,0 & $6,34 \mathrm{E}-03$ & $1,27 \mathrm{E}-06$ & 81,9 \\
\hline & 4 & 36 & 90 & 4,5 & 7,13E-03 & 1,09E-06 & 84,5 \\
\hline & 0 & 40 & 100 & 5,0 & 7,92E-03 & $8,29 \mathrm{E}-07$ & 88,2 \\
\hline
\end{tabular}


A Figura 4 apresenta as curvas de Tafel obtidas para os sistemas SME-OCS contendo ou não o inibidor IST. Observou-se deslocamento do potencial de corrosão para valores mais positivos à medida que a concentração de cada sistema aumentava, tendo sido possível confirmar a eficiência dos sistemas SME-OCS1-IST, SME-OCS2 e SME-OCS2-IST como inibidores de corrosão. A curva de Tafel do sistema SME-OCS1 foi avaliada previamente. ${ }^{23}$
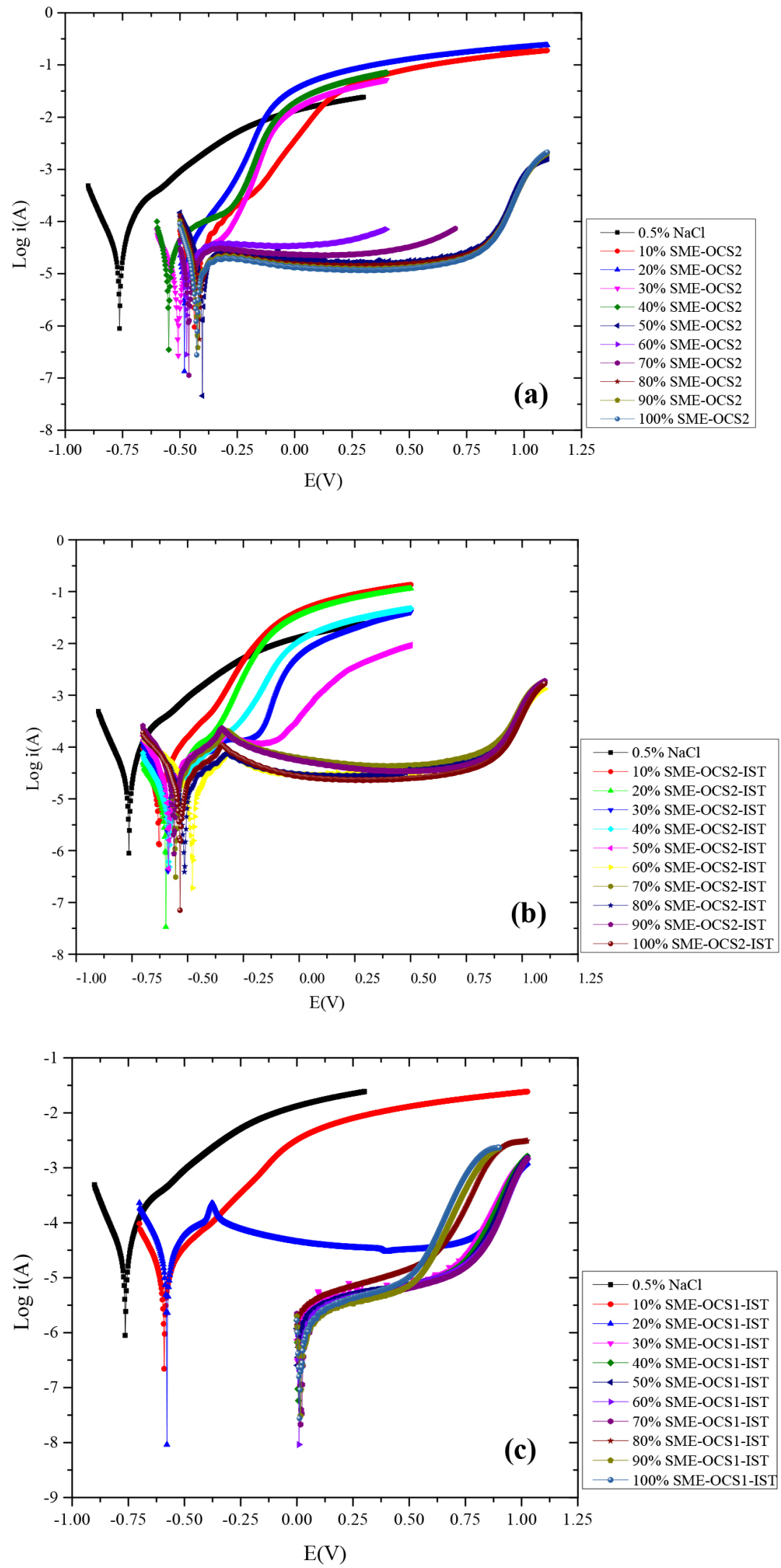

Figura 4. Curvas de Tafel: a) SME-OCS2; b) SME-OCS2-IST; c) SME-OCS1-IST 


\section{Isotermas de adsorção}

Com o objetivo de avaliar o processo de adsorção dos sistemas SME-OCS1-IST e SME-OCS2 sobre a superfície metálica foram aplicados três modelos de isotermas de adsorção: Langmuir, Frumkin e Temkin (Equações 7, 8 e 9, respectivamente). Os gráficos das isotermas foram traçados a partir da fração de recobrimento $(\theta)$, calculada para diferentes concentrações do tensoativo OCS nas microemulsões SME-OCS1 e SME-OCS2. As Figuras 1S - 9S (Material Suplementar) apresentam os gráficos das isotermas para os sistemas SME-OCS2, SME-OCS2-IST e SME-OCS1-IST, respectivamente.

$$
\begin{gathered}
\theta /(1-\theta)=K C \\
\log \theta /(1-\theta) C=\log K+g \theta \\
\log \theta / c=\log K+g \theta
\end{gathered}
$$

A análise das isotermas de adsorção mostrou que, para os sistemas SME-OCS2 e SME-OCS2-IST, a isoterma de adsorção que melhor se ajustou foi a isoterma de Langmuir, com fatores de correlação $\left(\mathrm{R}^{2}\right)$ 0,944 e 0,950 , respectivamente. No entanto, para SME-OCS1-IST, o melhor ajuste foi observado na isoterma de Frumkin $\left(\mathrm{R}^{2}=0,987\right)$, estando de acordo com resultado previamente observado para o processo de adsorção do sistema SME-OCS1, que também se ajusta à isoterma de adsorção de Frumkin. ${ }^{23}$

Através da Equação 10 realizou-se o cálculo da energia livre do processo de adsorção $\left(\Delta \mathrm{G}_{\mathrm{ads}}\right)$ para cada sistema microemulsionado, contendo ou não IST. O valor de 55,5 refere-se à concentração (mol L-1) da água. A Tabela 7 mostra os valores de $\Delta \mathrm{G}_{\mathrm{ads}}$ e das constantes de adsorção (K) obtidos.

$$
\mathrm{K}=(1 / 55,5) \exp \left(-\Delta \mathrm{G}_{\mathrm{ads}} / \mathrm{RT}\right)
$$

A partir das isotermas foram obtidas as constantes de adsorção (K) correlacionadas com o tensoativo OCS presente em cada sistema microemulsionado (SME-OCS), contendo ou não isatina (Tabela 7). De acordo com os dados da Tabela 7, observa-se que a constante de adsorção do sistema SME-OCS2-IST $(0,071)$ foi menor do que o valor do sistema veiculador SME-OCS2 $(0,560)$. Resultado similar é observado para as microemulsões SME-OCS1-IST $(0,382)$ e SME-OCS1 $(3,0)$. A partir dos valores de $\Delta \mathrm{G}_{\mathrm{ads}}$, concluiu-se que o processo de adsorção na interface líquido/sólido para todos os sistemas SME-OCS é espontâneo, devido aos valores negativos obtidos. A comparação efetuada para as energias livres de adsorção entre os sistemas veiculadores SME-OCS1 $(-12,67)$ e SME-OCS2 $(-8,51)$ revelou que as microemulsões contendo IST [SME-OCS1IST $(-7,57)$ e SME-OCS2-IST $(-3,39)]$ apresentam decréscimo nos valores modulares de $\Delta \mathrm{G}_{\mathrm{ads}}$.

Os sistemas isentos de IST (Tabela 7) apresentaram adsorção tipicamente física, ou seja, os efeitos entre o metal e o inibidor OCS são relativamente fracos. No entanto, as moléculas do tensoativo OCS exercem uma barreira de proteção no eletrodo, controlando o processo de corrosão. Apenas para o sistema SME-OCS2-IST $\left(\Delta \mathrm{G}_{\text {ads }}\right.$ $=-26,8 \mathrm{KJ} / \mathrm{mol}$ ) observa-se adsorção química.

Tabela 7. Parâmetros físico-químicos do tensoativo OCS em cada sistema SME-OCS

\begin{tabular}{lcc}
\hline SISTEMA & $\mathrm{K}_{\mathrm{ads}}\left(\mathrm{L} \mathrm{mol}^{-1}\right)$ & $\Delta \mathrm{G}_{\mathrm{ads}}\left(\mathrm{kJ} \mathrm{mol}^{-1}\right)$ \\
\hline SME-OCS1 & 3,00 & $-12,67$ \\
SME-OCS2 & 0,56 & $-8,51$ \\
SME-OCS1-IST & 82,47 & $-20,88$ \\
SME-OCS2-IST & 899,30 & $-26,80$ \\
\hline
\end{tabular}

Diferente do observado para o sistema SME-OCS2-IST (12,5\% de OCS), a energia livre de adsorção |20,88| para a microemulsão SME-OCS1-IST é característica de adsorção física. A justificativa pode estar correlacionada com uma suposta competição entre as moléculas do tensoativo OCS e da IST na ocupação dos sítios livres do metal, já que no sistema SME-OCS1 o tensoativo OCS está presente em maior percentual (20\% de OCS).

O fato do heterociclo IST não ser solúvel em meio salino, impossibilitou a realização de testes com esta substância não veiculada, o que dificulta um maior esclarecimento sobre sua eficiência de adsorção. No entanto, estudos preliminares comprovam sua eficiência (94\%) na inibição à corrosão de cobre, em meio ácido. ${ }^{12}$

A seguir encontra-se descrito um estudo computacional que foi realizado objetivando avaliar os efeitos estruturais da IST na inibição à corrosão.

\section{Cálculos computacionais e modelos de mecanismo de interação IST-metal}

A química teórica tem se mostrado altamente relevante no entendimento dos mecanismos de inibição à corrosão em diversos meios corrosivos. ${ }^{29,30}$ Neste contexto, foi realizado um estudo teórico quântico para investigação das interações entre o inibidor IST e a superfície metálica do aço carbono AISI 1020. O método empregado foi o B3LYP combinado com o conjunto de bases 6-311++G(d,p). Inicialmente, foram otimizados os parâmetros estruturais e eletrônicos da IST, juntamente com os cálculos das distribuições dos orbitais moleculares de fronteira, HOMO e LUMO, sob a estrutura do ligante IST (Figura 5 e Tabela 8). Estruturalmente, a IST é um composto planar contendo um sistema $\pi$-conjugado que compreende a molécula como um todo, que favorece seu processo de adsorção à superfície metálica.

Os valores das cargas verificados para os átomos de oxigênio O1 e O2, nitrogênio N3 e do carbono C4 são negativos (Tabela 8), estando coerente com o perfil de distribuição eletrônica da IST e pode ser verificado através da representação da superfície de potencial eletrostático (Figura 5b). A densidade eletrônica acentuada desta porção da molécula é decorrente tanto da natureza dos átomos de oxigênio e nitrogênio, quanto dos efeitos de ressonância típicos de compostos aromáticos e heteroaromáticos, os quais geram, de forma dinâmica, espécies carregadas que interagem mais fortemente com o sítio metálico. Este efeito é potencializado especialmente quando a IST faz parte do sistema microemulsionado de menor polaridade (SME-OCS1-IST), o qual apresentou eficiência à corrosão muito mais acentuada que o sistema de maior polaridade (SME-OCS2-IST).

A partir dos cálculos computacionais foi possível obter os valores das energias dos orbitais moleculares de fronteira, $E_{\text {номо }}$ e $E_{L U M O}$, que se encontram distribuídos por toda a estrutura da IST (Figuras $5 \mathrm{c}$ e 5d), cujos valores foram $-6,9592 \mathrm{eV}$ e $-3,1075 \mathrm{eV}$, respectivamente. Estes valores estão de acordos com aqueles encontrados teoricamente para outros inibidores de corrosão em aço carbono. ${ }^{28}$ Os valores de $\mathrm{E}_{\text {номо }}$ obtido, assim como $\Delta \mathrm{E}_{\text {gap }}=3,8517 \mathrm{eV}$, são coerentes com a capacidade do ligante IST interagir com os orbitais d vazios do metal, formando complexos estáveis, ${ }^{28}$ justificando, assim, a quimiossorção verificada experimentalmente. Por fim, a fração calculada de elétrons transferidos do inibidor para a superfície metálica foi 0,5106 , sendo este valor também consistente com uma situação de doação efetiva de elétrons do inibidor IST para a superfície do aço carbono.

\section{CONCLUSÃO}

As eficiências de inibições à corrosão foram avaliadas em aço carbono AISI 1020, em meio salino, para o heterociclo isatina (IST) 
(a)

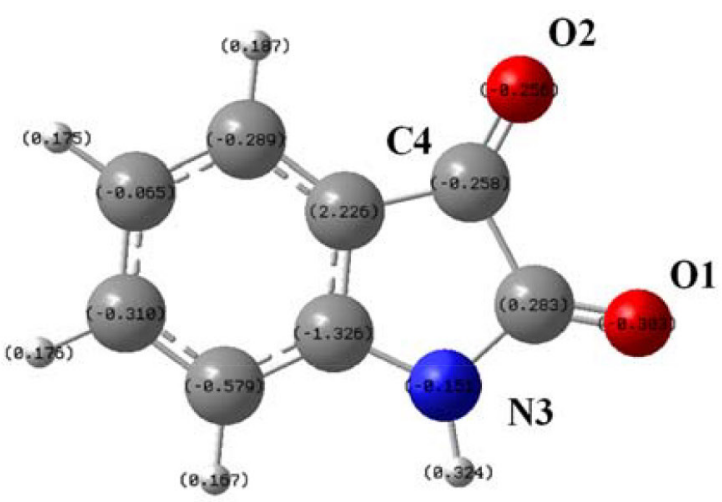

(c)

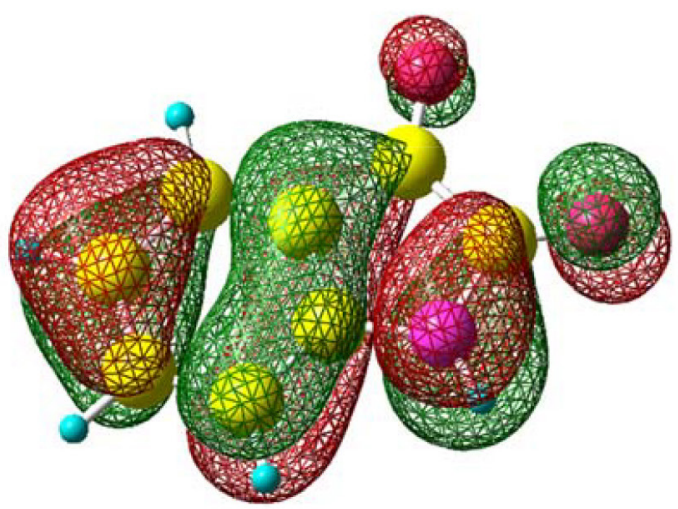

(b)

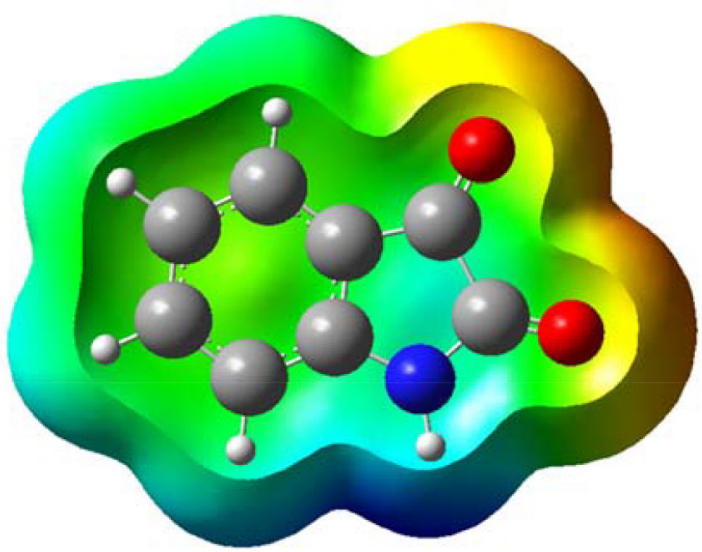

(d)

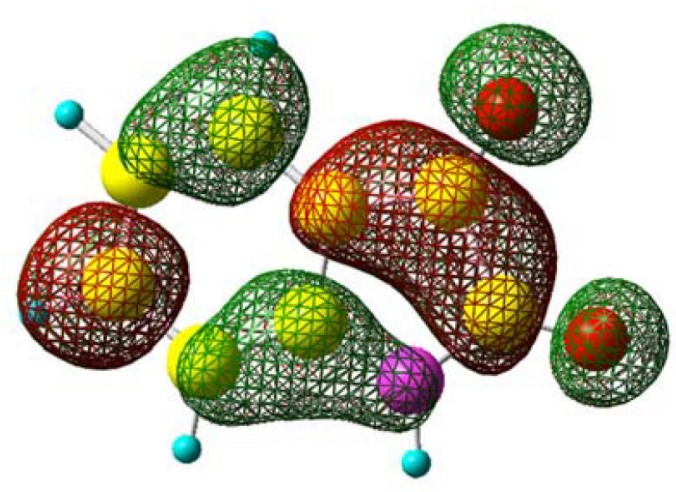

Figura 5. Resultados quânticos calculados para a IST utilizando o método DFT/B3LYP/6-311++G(d,p): (a) estrutura otimizada; (b) potencial eletrostático; (c) distribuição do HOMO; (d) distribuição do LUMO

Tabela 8. Parâmetros quânticos obtidos para a IST usando B3LYP/ 6-311++G(d,p) em fase gasosa

\begin{tabular}{lc}
\hline Parâmetro calculado para IST & Valor \\
\hline$E_{\text {номо }}(\mathrm{eV})$ & $-6,9592$ \\
$E_{\text {LUмо }}(\mathrm{eV})$ & $-3,1075$ \\
$\Delta \mathrm{E}_{\text {gap }}(\mathrm{eV})$ & 3,8517 \\
$\chi(\mathrm{eV})$ & 5,0333 \\
$\eta(\mathrm{eV})$ & 1,9258 \\
$\Delta N$ & $-0,5106$ \\
$\mu($ Debye $)$ & $-2,5166$ \\
Massa molecular (uma) & 147,03 \\
Carga em O1 & $-0,303$ \\
Carga em O2 & $-0,256$ \\
Carga em N3 & $-0,151$ \\
Carga em C4 & $-0,258$ \\
\hline
\end{tabular}

veiculado em baixas concentrações $\left(3,71 \times 10^{-2} \mathrm{mg}-7,92 \times 10^{-3} \mathrm{mg}\right)$ em dois sistemas microemulsionados contendo o tensoativo óleo de coco saponificado (OCS). O estudo de adsorção dos sistemas SMEOCS1-IST e SME-OCS2-IST avaliado em meio aquoso e salino $(\mathrm{NaCl}$ $0,5 \%)$, na interface líquido/gás, mostrou que a presença do íon $\left(\mathrm{Cl}^{-}\right)$ não ocasiona modificação na formação das micelas. De acordo com os valores observados para a energia livre de micelização $\left(\Delta \mathrm{G}_{\text {mic }}\right)$, observou-se a ocorrência de processo espontâneo de micelização.
As eficiências de inibição à corrosão das microemulsões SMEOCS1 e SME-OCS2 (sistemas veiculadores isentos de isatina), bem como SME-OCS1-IST e SME-OCS2-IST (sistemas contendo IST), foram avaliadas utilizando-se a técnica de voltametria de varredura linear (LPR), via método galvanostático, em que se obtém pontualmente, a linearidade de corrente.

Os cálculos das eficiências máximas de inibição foram realizados a partir das curvas de Tafel. O decréscimo da corrente e o deslocamento do potencial de corrosão para valores mais positivos confirmam a ação inibidora destas microemulsões. Comparativamente, o sistema SME-OCS1-IST apresentou melhor poder de inibição com eficiência máxima de 97,8\%. Para os sistemas SME-OCS2 e SME-OCS2-IST, a isoterma de adsorção que melhor se ajustou foi a isoterma de Langmuir, enquanto que para os sistemas SME-OCS $1^{23}$ e SMEOCS1-IST o melhor ajuste foi observado na isoterma de Frumkin.

Os valores da energia livre de adsorção foram negativos para todos os sistemas avaliados, indicando que o processo de adsorção na interface líquido/sólido é espontâneo. De acordo com os valores de $\Delta \mathrm{G}_{\text {ads }}$ observou-se que as microemulsões isentas de isatina (SMEOCS1 e SME-OCS2) apresentam adsorção tipicamente física e, neste caso, as moléculas do tensoativo OCS promovem uma barreira de proteção no eletrodo, controlando desta forma o processo de corrosão.

Para os sistemas com incorporação de IST, observou-se adsorção mista em que SME-OCS2-IST apresenta adsorção química e SME-OCS1-IST adsorção física, em função do maior percentual do tensoativo OCS (20\%). Esta diferença pode ser justificada pela redução da concentração do tensoativo OCS $(12,5 \%)$ na microemulsão SME-OCS2-IST, sugerindo que no processo de adsorção deste sistema prevalecem as características estruturais do heterociclo IST, 
tendo sido observado um $\Delta \mathrm{G}_{\mathrm{ads}}=-26,8 \mathrm{~kJ} / \mathrm{mol}$. Para o SME-OCS1IST as características estruturais predominantes foram do tensoativo OCS, cujo $\Delta \mathrm{G}_{\mathrm{ads}}=-20,88 \mathrm{~kJ} / \mathrm{mol}$.

Os estudos computacionais realizados suportam os resultados experimentais no que se refere à capacidade do heterociclo IST de se adsorver quimicamente à superfície do aço carbono, especificamente quando veiculado no sistema que contém menor percentual do tensoativo (SME-OCS2). Os valores de $E_{\text {номо }}$, de $\Delta \mathrm{E}_{g a p}$ e doação efetiva de elétrons do inibidor IST para a superfície metálica foram coerentes com a capacidade do ligante IST interagir com os orbitais d vazios do metal, formando complexos estáveis.

Com relação ao aspecto da adsorção é possível concluir que as curvas de Tafel são limitadas a uma região restrita de varredura de potencial, tendo sido observado que os potenciais são positivos. Desta forma, pode ser estimado um comportamento anódico para todos os formulados avaliados. Para continuidade desta pesquisa, portanto, pretende-se manter o meio salino e o tipo de veiculação (SME-OCS1IST e SME-OCS2-IST) para avaliação do comportamento do inibidor IST via processo inverso, ou seja, obtém-se a linearidade de potencial, para achar a corrente. Desta forma, o estudo poderá ser aprofundado via novos estudos de LPR e Impedância Eletroquímica.

De forma abrangente, a referida pesquisa está inserida em um estudo multidisciplinar, com aplicabilidade voltada para a eficácia de inibição à corrosão do composto orgânico IST. De fato, envolve o desenvolvimento de um novo "produto verde" que se mostrou eficaz no combate à corrosão $(97,7 \%$ de inibição para SME-OCS1-IST e $88,2 \%$ para SME-OCS20-IST). Este composto químico (isatina, IST) é amplamente comercializado (com aplicações diversas) e, no presente trabalho, por ter sido veiculado em baixas concentrações em um sistema biotecnológico microestruturado a base de óleo de coco saponificado (aproveitamento de matéria regional de baixo custo), poderá despertar a atenção da indústria química e, de fato, tornar-se um produto anticorrosivo $100 \%$ fabricado no país.

\section{MATERIAL SUPLEMENTAR}

As análises físico-químicas do óleo de coco, as medidas de viscosidade e de tensão superficial dos sistemas microemulsionados e as isotermas de adsorção (langmuir, Frumkim e Temkin) para os sistemas estudados estão disponíveis em http://quimicanova.sbq.org. br, na forma de arquivo PDF, com acesso livre.

\section{AGRADECIMENTOS}

À CAPES pelo o apoio financeiro deste trabalho. Ao Professor Dr. Angelo da Cunha Pinto ${ }^{\dagger}$ (In memoriam) pela disponibilização da amostra de isatina.

\section{REFERÊNCIAS}

1. Finšgar, M.; Jackson, J.; Corros. Sci. 2014, 86, 17.

2. Gutiérrez, E.; Rodríguez, J. A.; Cruz-Borbolla, J.; Alvarado-Rodríguez, J. G.; Thangarasu, P.; Corros. Sci. 2016, 108, 23.

3. Chigondo, M.; Chigando, F.; Journal of Chemistry 2016, ID 6208937, doi:10.1155/2016/6208937.

4. Qian, B.; Hou, B.; Zheng, M.; Corros. Sci. 2013, 72, 1.

5. Malik, M. A.; Hashim, M. A.; Nabi, F.; Al-Thabaiti, S. S.; Khan, Z.; Int. J. Electrochem. Sci. 2011, 6, 1927.

6. Jawich, M. W. S.; Oweimreen, G. A.; Ali, S. A.; Corros. Sci. 2012, 65,104 .
7. Prabha, S. S.; Rathish, R. J.; Dorothy, R.; Brindha, G.; Pandiarajan, M.; Al-Hashem, A.; Rajendran, S.; Eur. Chem. Bull. 2014, 3, 300.

8. Behzadnasab, M.; Mirabedini, S. M.; Kabiri, K.; Jamali, S. Corros. Sci. 2011, 53, 89.

9. Qui, L.; Xie, A.; Shen, Y.; Corros. Sci. 2005, 47, 273.

10. El-Achouri, M.; Infante, M. R.; Izquierdo, F.; Kertit, S.; Gouttaya, H. M.; Nciri, B.; Corros. Sci. 2001, 43, 19.

11. Hamid, Z. A.; Soror, T. Y.; El-Dahan, H. A.; Omar, A. M. A.; Anti-Corros. Meth. Mat. 1998, 45, 306.

12. Quartarone, G.; Bellomi, T.; Zingales, A.; Corros. Sci. 2003, 45, 715.

13. Singh, M. M.; Rastogi, R. B.; Upadhyai, B. N.; Corrosion 1994, 50, 620.

14. Abboud, Y.; Abourriche, A.; Saffaj, T.; Berrada, M.; Charrouf, M.; Bennamara, A.; Himidi, N. A.; Hannache, H.; Mat. Chem. Phys. 2007, 105, 1.

15. Finšgar, M.; Petovar, B.; Xhanari, K.; Maver, U.; Corros. Sci. 2016, 111, 370.

16. Silva, J. F. M.; Garden, S. J.; Pinto, A. C.; J. Braz. Chem. Soc. 2001, 12, 273.

17. Singh, D. D. N.; Singh, M. M.; Chaudhary, R. S.; Agarwal, C. V.; J Appl. Electrochem. 1980, 10, 587.

18. Ahamad, I.; Prasad, R.; Quraishi, M. A.; Corros. Sci. 2010, 52, 1472.

19. Ansari, K. R.; Quraishi, M. A.; Singh, A.; Corros. Sci. 2015, 95, 62.

20. Schwuger, M. J.; Schomäcker, R.; Chem. Rev. 1995, 95, 849.

21. Mendonça, C. R. B.; Bica, C. I. D.; Piatnicki, C. M. S.; Simó-Alfonso, E. F.; Ramis-Ramos, G.; Electrophoresis 2005, 26, 858.

22. Dantas T. N. C.; Silva, A. C.; Dantas Neto, A. A.; Fuel 2001, 80, 75.

23. Rossi, C. G. F. T.; Scatena Júnior, H.; Maciel, M. A. M.; Dantas, T. N. C.; Quim. Nova 2007, 30, 1128.

24. Moura, E. C. M.; Souza, A. D. N.; Rossi, C. G. F. T.; Silva, D. R.; Maciel, M. A. M.; Echevarria, A.; Bellieny, M. S. S.; Quim. Nova 2013, 36, 59.

25. Frisch, M. J.; Trucks, G. W.; Schlegel, H. B.; Scuseria, G. E.; Robb, M. A.; Cheeseman, J. R.; Montgomery, Jr., J. A.; Vreven, T.; Kudin, K. N.; Burant, J. C.; Millam, J. M.; Iyengar, S. S.; Tomasi, J.; Barone, V.; Mennucci, B.; Cossi, M.; Scalmani, G.; Rega, N.; Petersson, G. A.; Nakatsuji, H.; Hada, M.; Ehara, M.; Toyota, K.; Fukuda, R.; Hasegawa, J.; Ishida, M.; Nakajima, T.; Honda, Y.; Kitao, O.; Nakai, H.; Klene, M.; Li, X.; Knox, J. E.; Hratchian, H. P.; Cross, J. B.; Bakken, V.; Adamo, C.; Jaramillo, J.; Gomperts, R.; Stratmann, R. E.; Yazyev, O.; Austin, A. J.; Cammi, R.; Pomelli, C.; Ochterski, J. W.; Ayala, P. Y.; Morokuma, K.; Voth, G. A.; Salvador, P.; Dannenberg, J. J.; Zakrzewski, V. G.; Dapprich, S.; Daniels, A. D.; Strain, M. C.; Farkas, O.; Malick, D. K.; Rabuck, A. D.; Raghavachari, K.; Foresman, J. B.; Ortiz, J. V.; Cui, Q.; Baboul, A. G.; Clifford, S.; Cioslowski, J.; Stefanov, B. B.; Liu, G.; Liashenko, A.; Piskorz, P.; Komaromi, I.; Martin, R. L.; Fox, D. J.; Keith, T.; Al-Laham, M. A.; Peng, C. Y.; Nanayakkara, A.; Challacombe, M.; Gill, P. M. W.; Johnson, B.; Chen, W.; Wong, M. W.; Gonzalez, C.; Pople, J. A.; Gaussian, Inc., Wallingford CT, 2004.

26. Tirado-Rives, J.; Jorgensen, W. L.; J. Chem. Theory Comput. 2008, 4, 297.

27. Breneman, C. M.; Wiberg, K. B.; J. Comput. Chem. 1990, 11, 361.

28. Daoud, D.; Douadi, T.; Hamani, H.; Chafaa, S.; Al-Noaimi, M.; Corros. Sci. 2015, 94, 21.

29. Rochdi, A.; Kassou, O.; Dkhireche, N.; Touir, R.; Bakri, M. E.; Touhami, M. E.; Sfaira, M.; Mernari, B.; Hammouti, B.; Corros. Sci. 2014, 80, 442.

30. Sastri, V.S.; Perumareddi, J. R.; Corrosion 1997, 53, 617. 